\title{
HCC diagnosis DFP report
}

Accepted: 18 November 2020

(C) Springer Science+Business Media, LLC, part of Springer Nature 2021

\section{Members}

The membership of the 2020 HCC Diagnosis DFP includes: Khaled Elsayes (co-chair), Robert Marks (cochair), Alice Fung, Jimmy Lee, Kadar Jambhekar, Sandeep Deshmukh, Ryan Ash, Bijan Bijan, Venkateswar Surabhi, Irene Cruite, Alessandro Furlan, Joseph Yacoub, Sandy Lall, Anuradha Shenoy-Bangle, Zahra Kassam, Christopher Lim, William Masch, Shuchi Rogers, Amir Borhani, and Humaira Chaundry. Members-in-training include: Satheesh Krishna, Katie Blair, Mohab Elmohr, and Nikita Consul. Ex-officio members include: Claude Sirlin (founder and previous co-chair), Ania Kielar (previous co-chair), Victoria Chernyak, Richard Do, Kathryn Fowler, John McGahan, Mustafa Bashir, Elizabeth Hecht, and Aya Kamaya.

\section{Looking back}

After 6 years of hard work leading our DFP as the co-chair, Dr. Ania Kielar stepped down and turned over the leadership duties to the new co-chair, Dr. Robert Marks. Dr. Khaled Elsayes continued on as the co-chair as he began his second term of leadership.

At the Society of Abdominal Radiology 2020 Annual Scientific Meeting in Maui, the DFP presented our first handson workshop entitled "LI-RADS Cases for the Experts" presented by: Khaled Elsayes, Robert Marks, Victoria Chernyak, Irene Cruite, Sandeep Deshmukh, Richard Do, Katie Blair, Alice Fung, Aya Kamaya, and James Lee.

Members of the DFP also presented five separate workshops related to HCC diagnosis, including:

a. Overview of LI-RADS v2018. Presenters: Ania Kielar and Joseph Yacoub

b. LI-RADS v2018-Management Recommendations and Evaluation of Treatment Response. Presenters: Elizabeth Hecht and Mishal Mendiratta-Lala

c. Pitfalls of Applying LI-RADS; User and System Pitfalls. Presenters: Khaled Elsayes and Kathryn Fowler d. Liver Tumors in Non-LIRADS Populations. Presenters: Richard Do and Scott Gerst

e. LI-RADS Past, Present, and Future SAM session: Presenters: Victoria Chernyak, Robert Marks, William Masch, and Claude Sirlin

In response to the SARS-CoV-2 world-wide pandemic, as multiple educational activities were canceled, multiple members of the DFP, under the vision and leadership of Dr. Khaled Elsayes, took part in the Online Liver Imaging Course (OLIC), which was created to provide free, high quality education, accessible around the world on the topic of liver imaging. Each lecture lasted 60-80 min with time for live questions from the participants after each lecture. The OLIC was held from April 20, 2020 to May 29, 2020, with talks every weekday during the 6 week course. Each talk was uploaded and is available on YouTube (https://www. youtube.com/playlist?list=PLdZGE8Mpt7-1vYUtY_5Duii 6-zj4psGxh).

Over 1400 people world-wide registered for the OLIC. A post-course survey was sent to all registrants with 487 respondents to the survey. The results of the survey were published [1] and highlights from the survey include:

a. On average, there were 89 live participants per session and 750 YouTube views per recording

b. $97 \%$ of the respondents found the webinar series to be beneficial

c. Essentially all attendees felt that the webinar sessions met $(43 \%)$ or exceeded $(57 \%)$ their expectations.

d. $61 \%$ enjoyed the virtual format more than in-person conferences, while $38 \%$ preferred in-person conferences

e. $84 \%$ said they would attend future virtual conferences even if in-person conferences resume

Additionally, multiple members of the DFP published many review and original research articles regarding the diagnosis of HCC in the past 12 months. Twenty-three separate manuscripts were published in this time frame, a few including: 
a. LI-RADS Past, Present, and Future [2] and

b. Clinicians and Surgeon Survey Regarding Current and Future Versions of CT/MRI LI-RADS [3]

\section{Looking ahead}

The HCC Diagnosis DFP continues to have multiple educational and research projects upcoming in the next year. Twelve different educational exhibits on HCC diagnosis and LI-RADS were accepted and submitted at the 2020 RSNA Annual Meeting by members of the DFP. Online education continues to be a major focus of the DPF, and we will host a SAR HCC Diagnosis DFP Multidisciplinary Webinar in 2021. Additionally, due to the success of the OLIC, Dr. Khaled Elsayes has founded the Academy of Online Radiology Education (ACORE), and many members of the DFP are senior and guest editors of Liver Imaging for ACORE, producing lectures and blogs regarding liver imaging.

Additionally, as the Clinicians and Surgeons Survey on LI-RADS was published this year, a vast majority of the respondents were from academic centers. We plan on repeating and updating the study, however focusing on community abdominal imagers, clinicians, and surgeons, to understand their views about LI-RADS. Additionally, the DFP would like to increase its community radiologist membership to have more insight on the views and needs of the community radiologists regarding HCC diagnosis. Finally, we are planning more LI-RADS workshops for the SAR Annual Virtual Meeting 2021.

\section{References}

1. Elsayes KM, Marks RM, Kamel S, Towbin AJ, Kielar AZ, Patel P, et al. Online Liver Imaging Course; Pivoting to Transform Radiology Education During the SARS-CoV-2 Pandemic. Academic Radiology. 2020. doi: https://doi.org/10.1016/j.acra.2020.10.001.

2. Marks RM, Masch WR, Chernyak V. LI-RADS Past, Present, and Future, From the AJR Special Series on Radiology Reporting and Data Systems. AJR Am J Roentgenol. 2020. doi: https://doi. org/10.2214/AJR.20.24272.

3. Alenazi AO, Elsayes KM, Marks RM, Yacoub JH, Hecht EM, Chernyak V, et al. Clinicians and surgeon survey regarding current and future versions of CT/MRI LI-RADS. Abdom Radiol (NY). 2020. doi: https://doi.org/10.1007/s00261-020-02544-0.

Publisher's Note Springer Nature remains neutral with regard to jurisdictional claims in published maps and institutional affiliations. 